\title{
First-order aspects of tree paths
}

\author{
RUAan KelleRman \\ Department of Mathematics and Applied Mathematics, \\ University of Pretoria, Pretoria, South Africa \\ Email: ruaan.kellerman@up.ac.za
}

May 24, 2015

\begin{abstract}
Tree paths are investigated using first-order logic. The following results are obtained: (i) every definable path can be defined by a firstorder formula using at most one parameter chosen from the path itself; (ii) a canonical representation of the formulas that define definable paths is obtained; and (iii) every tree that has only finitely many paths that are not definable is $n$-equivalent to a tree of which all paths are definable. Moreover, a certain property that might be expected to hold, involving the transfer of $n$-equivalence between trees, is shown not to be true.
\end{abstract}

Keywords: tree, first-order, definable, path

\section{Introduction}

A systematic analysis of the first-order theories of trees, as done in [12] for linear orders, does not exist. It is known by Rabin's Tree Theorem $([11,9])$ that the monadic second-order theory of rooted binary trees with infinite paths is decidable and this result can be extended to other classes of trees. In [10], $n$-equivalence of finite coloured linear orders is investigated. Adding colours to linear orders increases the complexity of their analysis substantially. Hence a general analysis of the first-order theories of trees is likely to be complex: by partitioning a tree along any of its paths and assigning to each part in this partition a first-order sentence of some fixed quantifier rank that characterises that part of the tree, one obtains a coloured linear order. The analysis of the first-order theories of trees is further complicated by the fact 
that not all paths in a tree are definable and one cannot quantify over individual paths. Trees may contain so-called emergent paths (see $[8,7])$ that have the property that every node in the path belongs to infinitely many other paths too, thus hindering attempts to impose particular structural properties on paths in a discriminate manner.

Some important known results about the first-order theories of trees include: the first-order theory of the class of finite trees given in [1], the first-order theory of the class of well-founded trees (trees with a well-founded prefix ordering) given in [2], and that every tree is $n$-equivalent, for suitable $n$, to a finitely branching tree $([13,6])$. In [7] the relationship between a class of linear orders $\mathcal{C}$, and the corresponding class $\mathcal{T}(\mathcal{C})$ of trees having paths that are isomorphic to linear orders in $\mathcal{C}$, is investigated. In [5] axiomatisations of the monadic second order theory, the first-order logic with transitive closure theory, and the first-order logic with least fixed points theory, of finite trees, is given.

The structure of a tree is determined by the order types of its paths and by its branching behaviour. There are subtle relationships between these two structural properties: consider for example König's Lemma (a well-founded tree of height $\omega$ has a path with order type $\omega$ if it is finitely branching) and the existence of Suslin trees (wellfounded trees of height $\omega_{1}$ that do not contain any paths with order type $\omega_{1}$ and that do not have any uncountable antichains) which is undecidable within ZFC.

This paper examines some first-order aspects of paths in trees. A broad set-theoretical definition of trees is used: trees are not required to be finite, rooted, discrete, finitely branching, or well-founded. The paper is structured as follows:

- Section 2: Background. Some terminology and notation that is used later is fixed. A short overview of relativisations and characteristic sentences is given.

- Section 3: Trees. Trees are defined set-theoretically. Paths and path defining formulas are introduced. Some tree composition results are given.

- Section 4: Canonical form of formulas that define paths. A lemma dealing with the elimination of parameters from trees is given. This lemma is used to prove the following result: every definable path can be defined by a first-order formula using at most one parameter chosen from the path itself. A canonical representation of the first-order formula that defines a definable path is given.

- Section 5: Trees that have only finitely many undefin- 
able paths. The transfer of truth of first-order sentences between a tree and certain of its subtrees is investigated. It is shown that, for every natural number $n$, every tree that has at most finitely many paths that are not definable is $n$-equivalent to a tree of which all paths are definable.

- Section 6: The partition and cover properties. Using a Ramsey-type theorem, a proof of the following partition property is given: Every tree can, for any natural number $n$, be partitioned along any of its paths into subtrees all but one of which are pairwise $n$-equivalent. In contrast to this, a certain cover property that one might expect to be true, involving the transfer of first-order equivalence between trees with equivalent covers, is shown to be false. This cover property fails because end extensions of trees need not be elementary extensions. A further condition involving elementary chains that is sufficient for this cover property to hold is suggested.

The motivation behind the partition property and cover property introduced in Section 6 lies in the problem of axiomatising the firstorder theory of the class $\mathcal{T}_{\alpha}$ that consists of trees of which every path is isomorphic to the ordinal $\alpha$. Consider the case where $\alpha=\omega+1$. The first-order theory of $\mathcal{T}_{\omega+1}$ admits models that have paths that are not isomorphic to $\omega+1$. Consider for example a tree $\mathfrak{B}_{\omega+1}$ of which each path is isomorphic to $\omega+1$ and of which each non-leaf node has exactly two successors. Since $\mathfrak{B}_{\omega+1}$ has at least $2^{\aleph_{0}}$ many paths, and since each path has a leaf node, the cardinality of $\mathfrak{B}_{\omega+1}$ is at least $2^{\aleph_{0}}$ so by the downwards Löwenheim-Skolem theorem, $\mathfrak{B}_{\omega+1}$ has a countably infinite elementary substructure $\mathfrak{B}_{\omega+1}^{\prime}$. Each non-leaf node in $\mathfrak{B}_{\omega+1}^{\prime}$ also has exactly two immediate successors so $\mathfrak{B}_{\omega+1}^{\prime}$ will differ from $\mathfrak{B}_{\omega+1}$ only in the distribution of its leaf nodes. In particular, $\mathfrak{B}_{\omega+1}^{\prime}$ must contain paths that are isomorphic to $\omega$. The paths in $\mathfrak{B}_{\omega+1}^{\prime}$ that are parametrically definable are precisely the ones that are isomorphic to $\omega+1$ while the paths that are not parametrically definable are precisely the ones that are isomorphic to $\omega$. Since $\mathfrak{B}_{\omega+1} \in \mathcal{T}_{\omega+1}$ and $\mathfrak{B}_{\omega+1}^{\prime} \equiv \mathfrak{B}_{\omega+1}$ then $\mathfrak{B}_{\omega+1}^{\prime}$ is a model of the first-order theory of $\mathcal{T}_{\omega+1}$.

Now suppose we have a candidate set of axioms $A$ that we wish to show axiomatises the first-order theory of $\mathcal{T}_{\omega+1}$. One way to show that $A$ does indeed axiomatise the first-order theory of $\mathcal{T}_{\omega+1}$ would be to show, for each natural number $n$, that every model $\mathfrak{M}$ of $\mathrm{A}$ is $n$ equivalent to a tree in $\mathcal{T}_{\omega+1}$. The model $\mathfrak{M}$ may contain paths that are isomorphic to $\omega$ (as in the case of the tree $\mathfrak{B}_{\omega+1}^{\prime}$ above), so a possible strategy would be to show that each path in $\mathfrak{M}$ that is isomorphic to $\omega$ can be augmented with a leaf node to obtain a path that is isomorphic to $\omega+1$. The resulting tree will be a member of the class $\mathcal{T}_{\omega+1}$ and 
if it is $n$-equivalent to $\mathfrak{M}$ then we will have succeeded in showing that A axiomatises the first-order theory of $\mathcal{T}_{\omega+1}$.

The partition property and cover property involve the transfer of $n$-equivalence between trees in the context described above and may be useful in showing the completeness of a set of axioms for the firstorder theory of the class $\mathcal{T}_{\alpha}$.

\section{Background}

The reader is referred to [2] for more information about characteristic sentences, and to [12] for more information about relativisations. The details from $[2,12]$ that are relevant to this paper are briefly mentioned in the sections on characteristic sentences and relativisations below.

The axiom of choice is assumed.

\subsection{Some notation and terminology}

The concatenation of tuples $\bar{x}$ and $\bar{y}$ will be denoted as $\bar{x} \bar{y}$. The length of the tuple $\bar{x}=\left(x_{i}\right)_{i \in \alpha}$ is taken as the ordinal $\alpha$ and will be denoted as $\ell(\bar{x}) . \overline{0}_{\alpha}$ will denote the tuple of length $\alpha$ of which every entry is 0 and $\overline{1}_{\alpha}$ will denote the tuple of length $\alpha$ of which every entry is $1 . \varphi(u / x)$ denotes the formula $\varphi(x)$ with $u$ substituted for $x$. The quantifier rank of a formula $\varphi$ is denoted as $\operatorname{qr}(\varphi)$. The domain of a structure $\mathfrak{A}$ is denoted as $|\mathfrak{A}|$. Given a structure $\mathfrak{A}$ and a tuple $\bar{a}$ with entries from $|\mathfrak{A}|,(\mathfrak{A} ; \bar{a})$ denotes the structure $\mathfrak{A}$ with the elements in $\bar{a}$ added as constants. The first-order formula $\varphi(x, \bar{a})$ defines the set $B \subseteq|\mathfrak{A}|$ in the structure $(\mathfrak{A} ; \bar{a})$ when $u \in B$ if and only if $(\mathfrak{A} ; \bar{a}) \models \varphi(u / x, \bar{a})$. The set $B$ is definable in $\mathfrak{A}$ when there exists a tuple $\bar{a}$ of elements from $|\mathfrak{A}|$ and a first-order formula $\varphi(x, \bar{a})$ such that $\varphi(x, \bar{a})$ defines $B$ in $(\mathfrak{A} ; \bar{a})$. Two structures $\mathfrak{A}$ and $\mathfrak{B}$ are called $n$-equivalent, denoted $\mathfrak{A} \equiv_{n} \mathfrak{B}$, when $\mathfrak{A}$ and $\mathfrak{B}$ satisfy the same first-order sentences of quantifier rank less than or equal to $n$.

Ehrenfeucht-Fraïssé games (see e.g. [3]) will be used to show the $n$-equivalence of structures. An $n$-round Ehrenfeucht-Fraïssé game is played by two players - Player I and Player II - on structures $\mathfrak{A}$ and $\mathfrak{B}$, as follows. In each round $i$ of the game $(1 \leqslant i \leqslant n)$, Player I chooses an element from either of the two structures, say $a_{i} \in|\mathfrak{A}|$ (respectively $b_{i} \in|\mathfrak{B}|$ ), and Player II responds by choosing an element $b_{i} \in|\mathfrak{B}|$ (respectively $\left.a_{i} \in|\mathfrak{A}|\right)$. Let $\bar{a}=\left(a_{1}, \ldots, a_{n}\right)$ and $\bar{b}=\left(b_{1}, \ldots, b_{n}\right)$. Player II then wins the game if the structures $(\mathfrak{A} ; \bar{a})$ and $(\mathfrak{B} ; \bar{b})$ satisfy the same atomic sentences. Player II has a winning strategy for the 
$n$-round game on $\mathfrak{A}$ and $\mathfrak{B}$ (a strategy that allows her to win the game regardless how Player I chooses his elements) if and only if $\mathfrak{A} \equiv_{n} \mathfrak{B}$.

The rank of a formula $\varphi$ is the sum of $\operatorname{qr}(\varphi)$ and the number of free variables in $\varphi$. $\mathfrak{A}$ is called an $n$-elementary substructure of $\mathfrak{B}$, denoted $\mathfrak{A} \preceq_{n} \mathfrak{B}$, when $\mathfrak{A}$ is a substructure of $\mathfrak{B}$ and for every formula $\varphi(\bar{x})$ of rank at most $n$ where $\bar{x}$ is a $k$-tuple of variables, if $\bar{a} \in|\mathfrak{A}|^{k}$ then $\mathfrak{A}=$ $\varphi(\bar{a} / \bar{x})$ if and only if $\mathfrak{B} \models \varphi(\bar{a} / \bar{x})$. The Tarski-Vaught criterion for elementary substructures generalises to $n$-elementary substructures: if $\mathfrak{A}$ is a substructure of $\mathfrak{B}$ then $\mathfrak{A} \preceq_{n} \mathfrak{B}$ if and only if for every formula $\varphi(x, \bar{y})$ of rank at most $n$ and for every tuple $\bar{a}$ with entries from $|\mathfrak{A}|$, if $\mathfrak{B} \models \exists x \varphi(x, \bar{a} / \bar{y})$ then $\mathfrak{A} \models \exists x \varphi(x, \bar{a} / \bar{y})$. Moreover, if $\left\{\mathfrak{A}_{i}\right\}_{i \in \alpha}$ is a chain of structures such that $\mathfrak{A}_{i} \preceq_{n} \mathfrak{A}_{j}$ when $i<j$ then $\mathfrak{A}_{k} \preceq_{n} \bigcup_{i \in \alpha} \mathfrak{A}_{i}$ for all $k$.

The order types of $(\{0, \ldots, k-1\} ;<)$ and $(\mathbb{N} ;<)$ will be denoted as $\mathbf{k}$ and $\omega$ respectively. Ordinals will sometimes be identified with their domains and the finite ordinal $\mathbf{k}$ will sometimes be identified with the natural number $k$. Given a linear order $\mathfrak{L}=(L ;<)$ and an ordinal $\alpha$, an increasing sequence $\left(a_{i}\right)_{i \in \alpha}$ in $L$ is called cofinal in $\mathfrak{L}$ when, for every $x \in L$, there exist $j \in \alpha$ such that $x \leqslant a_{j}$. The least ordinal $\alpha$ for which a sequence $\left(a_{i}\right)_{i \in \alpha}$ that is cofinal in $\mathfrak{L}$ exists, is called the cofinality of $\mathfrak{L}$.

\subsection{Relativisations}

Let $\mathfrak{A}$ be any structure. Let $\bar{x}$ be an $n$-tuple of variables, $\bar{y}$ be a $k$ tuple of variables all of which are different from the variables in $\bar{x}$, and $\bar{a} \in|\mathfrak{A}|^{k}$ (the tuples $\bar{x}, \bar{y}$ and $\bar{a}$ may be empty).

For $\varphi(\bar{x})$ and $\theta(u, \bar{y})$ any first-order formulas, the relativisation of $\varphi$ to $\theta$ is denoted as $\varphi^{\theta}$ (where $\varphi^{\theta}=\varphi^{\theta}(\bar{x}, \bar{y})$ ) and is defined as follows:

- If $\varphi$ is atomic then $\varphi^{\theta}:=\varphi$.

- If $\varphi=\neg \psi$ then $\varphi^{\theta}:=\neg\left(\psi^{\theta}\right)$.

- If $\varphi=\psi_{1} \star \psi_{2}$, where $\star$ is any of the connectives $\vee, \wedge, \rightarrow$ or $\leftrightarrow$, then $\varphi^{\theta}:=\psi_{1}^{\theta} \star \psi_{2}^{\theta}$.

- If $\varphi=\exists x \psi$ then $\varphi^{\theta}:=\exists x\left(\theta(x, \bar{y}) \wedge \psi^{\theta}\right)$.

- If $\varphi=\forall x \psi$ then $\varphi^{\theta}:=\forall x\left(\theta(x, \bar{y}) \rightarrow \psi^{\theta}\right)$.

Define $(\mathfrak{A} ; \bar{a})^{\theta}$ to be the structure with domain

$$
\{z \in|\mathfrak{A}|:(\mathfrak{A} ; \bar{a}) \models \theta(z / u, \bar{a})\}
$$

and subject to the constant symbols, relation symbols, and function symbols of the structure $\mathfrak{A}$ but restricted to the set (1). 
Proposition $1([\mathbf{1 2}])$ Let $\varphi(\bar{x})$ and $\theta(u, \bar{y})$ be any first-order formulas. For any $n$-tuple of elements $\bar{b}$ from $\left|(\mathfrak{A} ; \bar{a})^{\theta}\right|$,

$$
\mathfrak{A} \models \varphi^{\theta}(\bar{b} / \bar{x}, \bar{a} / \bar{y}) \Leftrightarrow(\mathfrak{A} ; \bar{a})^{\theta} \models \varphi(\bar{b} / \bar{x}) .
$$

\subsection{Characteristic sentences}

Fix structures $\mathfrak{A}$ and $\mathfrak{B}$ with the same finite relational signatures. Let $\bar{a} \in|\mathfrak{A}|^{k}$ and $\bar{b} \in|\mathfrak{B}|^{k}$ and let $\bar{x}$ be a $k$-tuple of variables (the tuples $\bar{a}, \bar{b}$ and $\bar{x}$ may be empty).

For $n$ any natural number, the $n$-characteristic formula $\llbracket(\mathfrak{A} ; \bar{a}) \rrbracket^{n}$ (where $\left.\llbracket(\mathfrak{A} ; \bar{a}) \rrbracket^{n}=\llbracket(\mathfrak{A} ; \bar{a}) \rrbracket^{n}(\bar{x})\right)$ of the structure $\mathfrak{A}$ over the tuple $\bar{a}$ is defined as follows:

- $\llbracket(\mathfrak{A} ; \bar{a}) \rrbracket^{0}:=\bigwedge\{\varphi(\bar{x}): \varphi$ an atomic or negated atomic formula with $\mathfrak{A} \models \varphi(\bar{a} / \bar{x})\} ;$

- $\llbracket(\mathfrak{A} ; \bar{a}) \rrbracket^{m+1}:=\bigwedge_{a_{k+1} \in|\mathfrak{A}|} \exists x_{k+1} \llbracket\left(\mathfrak{A} ; \bar{a} a_{k+1}\right) \rrbracket^{m} \wedge$

$$
\forall x_{k+1} \bigvee_{a_{k+1} \in|\mathfrak{A}|} \llbracket\left(\mathfrak{A} ; \bar{a} a_{k+1}\right) \rrbracket^{m}
$$

For languages with finite relational signatures it can be shown that, for all natural numbers $n$ and $k$, there are only finitely many pairwise non-equivalent $n$-characteristic formulas, taken over the class of all structures in that signature and all $k$-tuples in those structures. If $\bar{a}$ is the empty tuple then $\llbracket(\mathfrak{A} ; \bar{a}) \rrbracket^{n}$ is written as $\llbracket \mathfrak{A} \rrbracket^{n}$ and is called the $n$-characteristic sentence of $\mathfrak{A}$.

Lemma 2 ([2]) The following hold for every natural number $n$ :

(i) $\mathfrak{A} \models \llbracket(\mathfrak{A} ; \bar{a}) \rrbracket^{n}(\bar{a} / \bar{x})$;

(ii) the formula $\llbracket(\mathfrak{A} ; \bar{a}) \rrbracket^{n}$ has quantifier rank $n$.

Theorem 3 ([2]) The following statements are equivalent for every natural number $n$ :

(i) $(\mathfrak{A} ; \bar{a}) \equiv_{n}(\mathfrak{B} ; \bar{b})$;

(ii) $\mathfrak{B} \models \llbracket(\mathfrak{A} ; \bar{a}) \rrbracket^{n}(\bar{b} / \bar{x})$;

(iii) the formulas $\llbracket(\mathfrak{A} ; \bar{a}) \rrbracket^{n}$ and $\llbracket(\mathfrak{B} ; \bar{b}) \rrbracket^{n}$ are equivalent.

\section{Trees}

\subsection{Trees as ordered sets}

The simplest first-order language for trees has no constant symbols, two relation symbols (the usual equality symbol $=$ and an order sym- 
bol $<$ ), and no function symbols. Define the following first-order formulas in this language:

$$
\begin{aligned}
x \smile y & :=x<y \vee x=y \vee y<x \\
x \leqslant y & :=x<y \vee x=y
\end{aligned}
$$

A tree is then a structure $\mathfrak{T}=(T ;<)$ that satisfies the following firstorder sentences:

- $\forall x \neg(x<x) \quad$ (irreflexivity);

- $\forall x \forall y \forall z(x<y \wedge y<z \rightarrow x<z) \quad$ (transitivity);

- $\forall x \forall y \forall z((y<x \wedge z<x) \rightarrow(y \smile z)) \quad$ (subtotalness);

- $\forall x \forall y \exists z(z \leqslant x \wedge z \leqslant y) \quad$ (connectedness).

The elements of $T$ are called nodes. Given a tuple $\bar{a}$ of nodes from $T$ and a subset $A \subseteq T$, the language may sometimes be enriched by adding the tuple of constant symbols $\bar{a}$ to obtain the structure $(\mathfrak{T} ; \bar{a})$, or by adding the unary relation symbol $A$ to obtain the structure $(\mathfrak{T} ; A)$, where for $u \in T$ it holds that $(\mathfrak{T} ; A) \models A(u / x)$ if and only if $u \in A$. When referring to a tree $\mathfrak{T}$ without specifying additional constant or relation symbols, it will be understood that $\mathfrak{T}$ has the form $\mathfrak{T}=(T ;<)$. A leaf is node that is maximal with respect to $<$.

Given nodes $a, b \in T$ with $a \leqslant b$, define the following sets:

$$
\begin{aligned}
a^{\leqslant} & :=\{x \in T: a \leqslant x\} \\
b^{>} & :=\{x \in T: x<b\} \\
C(b) & :=\{x \in T: b \nless x\} \\
T_{a}^{b} & :=a \leqslant \cap C(b) \\
{[a, b) } & :=\{x \in T: a \leqslant x<b\}
\end{aligned}
$$

Define the following trees:

$$
\begin{aligned}
\mathfrak{T}_{a} & :=(\mathfrak{T} ; a)^{a \leqslant x}=\left(a^{\leqslant} ;<\uparrow_{a} \leqslant\right) \\
\mathfrak{T}^{b} & :=(\mathfrak{T} ; b)^{\neg(b \leqslant x)}=\left(C(b) ;<\uparrow_{C(b)}\right) \\
\mathfrak{T}_{a}^{b} & :=(\mathfrak{T} ; a, b)^{a \leqslant x \wedge \neg(b \leqslant x)}=\left(T_{a}^{b} ;<\uparrow_{T_{a}^{b}}\right) \\
\star^{\mathfrak{T}^{b}} & :=\left(\mathfrak{T}^{b} ; b^{>}\right) \\
\star^{\mathfrak{T}_{a}^{b}} & :=\left(\mathfrak{T}_{a}^{b} ;[a, b)\right)
\end{aligned}
$$

Note that, for trees $\mathfrak{T}$ and $\mathfrak{S}$ with $a, b \in T$ such that $a<_{T} b$ and $c, d \in S$ such that $c<_{S} d$, if $\mathfrak{T}^{a} \equiv_{n} \mathfrak{S}^{c}$ and $\mathfrak{T}^{b} \equiv_{n} \mathfrak{S}^{d}$, it need not be the case that $\mathfrak{T}_{a}^{b} \equiv_{n} \mathfrak{S}_{c}^{d}$. 


\subsection{Paths}

Let $\mathfrak{T}=(T ;<)$ be a tree. If $A$ is a linearly ordered subset of $T$ that is maximal in the set of all linearly ordered subsets of $T$ then $\left(A ;<\uparrow_{A}\right)$ is called a path in $\mathfrak{T}$. As a notational convenience the path $\left(A ;<\uparrow_{A}\right)$ will usually simply be identified with the set $A$ although it must be understood that a path is a linear order rather than just a set.

For $k$ any natural number and $\varphi(x, \bar{z})$ any formula with $\bar{z}$ a $k$-tuple of variables different from $x$, define the formula

$$
\begin{aligned}
& \pi_{\varphi}(\bar{z}):=\exists x \varphi(x, \bar{z}) \wedge \forall x \forall y(\varphi(x, \bar{z}) \wedge \varphi(y, \bar{z}) \rightarrow(x \smile y)) \wedge \\
& \forall x \forall y(x<y \wedge \varphi(y, \bar{z}) \rightarrow \varphi(x, \bar{z})) \wedge \neg \exists x \forall y(\varphi(y, \bar{z}) \rightarrow y<x) .
\end{aligned}
$$

If $\bar{a}$ is a $k$-tuple consisting of nodes from $T$ then $\varphi(x, \bar{a})$ defines a path in $(\mathfrak{T} ; \bar{a})$ if and only if $(\mathfrak{T} ; \bar{a}) \models \pi_{\varphi}(\bar{a})$.

Let $\Sigma$ be a first-order theory. The tree $\mathfrak{T}$ will satisfy the theory

$$
\left\{\forall \bar{z}\left(\pi_{\varphi}(\bar{z}) \rightarrow \sigma^{\varphi}(\bar{z})\right): \varphi(x, \bar{z}) \text { is any formula and } \sigma \in \Sigma\right\}
$$

if and only if every definable path in $\mathfrak{T}$ satisfies the theory $\Sigma$.

A path $A$ is called singular when there exists $a \in A$ such that $A=\{x \in T: x \smile a\}$, else $A$ is called emergent. Every singular path is definable: if $A$ is singular with $A=\{x \in T: x \smile a\}$ then the formula $\varphi(x, a)=x \smile a$ defines $A$ in $(\mathfrak{T} ; a)$. If $B \subseteq A$ then $\left(B ;<\uparrow_{B}\right)$ is called a stem when the following condition is satisfied: if $x \in B$ and $y<x$ then $y \in B$ (in other words, stems are simply downwards closed subsets in a tree). Again the stem $\left(B ;<\uparrow_{B}\right)$ will usually simply be identified with the set $B$ although a stem is actually a linear order rather than just a set. Given a tree $\mathfrak{T}$ and a stem $B$ in $\mathfrak{T}$, define the following sets and trees $\left(X^{c}\right.$ denotes the set-theoretical complement of the set $X$ ):

$$
\begin{aligned}
B^{<} & :=\{x \in T: y<x \text { for all } y \in B\} \\
\mathfrak{T}^{B} & :=\left(\left(B^{<}\right)^{c} ;<\uparrow_{\left(B^{<}\right)^{c}}\right) \\
\star^{\mathfrak{T}^{B}} & :=\left(\mathfrak{T}^{B} ; B\right)
\end{aligned}
$$

Note that if $\left(b_{i}\right)_{i \in \beta}$ is a cofinal sequence of nodes in $B$ and $\beta$ is a limit ordinal then

$$
\bigcup_{i \in \beta} \mathfrak{T}^{b_{i}}={ }_{\star} \mathfrak{T}^{B}
$$




\subsection{Tree composition theorems}

The following Feferman-Vaught-type composition theorems will be used in the remainder of the paper. The first result, which is a refinement of the Feferman-Vaught composition theorem for ordinal sums (see [4]), can be proved using a straightforward application of Ehrenfeucht-Fraïssé games and the proof is omitted.

Lemma 4 Let $n$ be a natural number. Let $\mathfrak{T}=\left(T ;<_{T}\right)$ and $\mathfrak{S}=$ $\left(S ;<_{S}\right)$ be trees. Let $a, b, c \in T$ and $p, q, r \in S$ with $a<_{T} b<_{T} c$ and $p<_{S} q<_{S}$ r. If ${ }_{\star} \mathfrak{T}_{a}^{b} \equiv_{n \star} \mathfrak{S}_{p}^{q}$ and ${ }_{\star} \mathfrak{T}_{b}^{c} \equiv_{n \star} \mathfrak{S}_{q}^{r}$ then ${ }_{\star} \mathfrak{T}_{a}^{c} \equiv_{n \star} \mathfrak{S}_{p}^{r}$.

The following known result (see e.g. [3, Lemma 3.12]) is used in the proof of Lemma 5:

$$
\text { If } k, m \geqslant 2^{n}-1 \text { then } \mathbf{k} \equiv_{n} \mathbf{m} \text {. }
$$

Lemma 5 Let $n$ be a natural number. Let $\mathfrak{T}=\left(T ;<_{T}\right)$ and $\mathfrak{S}=$ $\left(S ;<_{S}\right)$ be any trees. Let $\tau$ and $\sigma$ be ordinals (possibly finite) such that $\tau \equiv_{n} \sigma$. Let $\left(a_{i}\right)_{i \in 1+\tau}$ and $\left(b_{i}\right)_{i \in 1+\sigma}$ be increasing sequences of nodes in $T$ and $S$ respectively such that ${ }_{\star} \mathfrak{T}_{a_{i}}^{a_{i+1}} \equiv_{n \star} \mathfrak{S}_{b_{j}}^{b_{j+1}}$ for all $i$ and $j$ with $i, i+1 \in 1+\tau$ and $j, j+1 \in 1+\sigma .{ }^{1}$ Let $A=\bigcup_{i \in 1+\tau}\left[a_{0}, a_{i}\right)$, $B=\bigcup_{i \in 1+\sigma}\left[b_{0}, b_{i}\right), A^{\prime}=\left\{x \in T: a_{i} \leqslant x\right.$ for all $\left.i\right\}, B^{\prime}=\left\{x \in S: b_{i} \leqslant\right.$ $x$ for all $i$,

$$
\begin{aligned}
& \mathfrak{T}^{\prime}=\left(a_{0}^{\leqslant} \backslash A^{\prime} ;\left(<_{T}\right) \uparrow_{\left(a_{0}^{\leqslant} \backslash A^{\prime}\right)}, A\right), \\
& \mathfrak{S}^{\prime}=\left(b_{0}^{\lessgtr} \backslash B^{\prime} ;\left(<_{S}\right) \uparrow_{\left(b_{0}^{\lessgtr} \backslash B^{\prime}\right)}, B\right) .
\end{aligned}
$$

Then $\mathfrak{T}^{\prime} \equiv_{n} \mathfrak{S}^{\prime}$.

Proof Given a node $t \in\left|\mathfrak{T}^{\prime}\right|$ (respectively $s \in\left|\mathfrak{S}^{\prime}\right|$ ), let $t^{\tau}$ (respectively $s^{\sigma}$ ) be the greatest element $i \in 1+\tau$ (respectively $i \in 1+\sigma$ ) such that $t \in a_{i}^{\leqslant}$(respectively $s \in b_{i}^{\leqslant}$). Note that $t \in T_{a_{t} \tau}^{a_{t} \tau+1}$ and $s \in S_{b_{s} \sigma}^{b_{s} \sigma}$ for all $t \in\left|\mathfrak{T}^{\prime}\right|$ and $s \in\left|\mathfrak{S}^{\prime}\right|$.

We describe a winning strategy for Player II for the $n$-round Ehrenfeucht-Fraïssé game on $\mathfrak{T}^{\prime}$ and $\mathfrak{S}^{\prime}$. We will play two sets of smaller Ehrenfeucht-Fraïssé games in parallel with the main game on $\mathfrak{T}^{\prime}$ and $\mathfrak{S}^{\prime}$, and the nodes that have been played in these smaller games in their earlier rounds will be determined by the nodes that have been played in the main game in its earlier rounds. The two sets of smaller games that we will consider are (i) the $n$-round game on $\tau$ and $\sigma$ where

\footnotetext{
${ }^{1}$ The first element in $1+\tau$ and $1+\sigma$ is taken as 0 . If $\tau$ and $\sigma$ are infinite then $1+\tau=\tau$ and $1+\sigma=\sigma$.
} 
the elements played in its earlier rounds are of the form $t^{\tau} \in \tau$ and $s^{\sigma} \in \sigma$ for the nodes $t \in\left|\mathfrak{T}^{\prime}\right|$ and $s \in\left|\mathfrak{S}^{\prime}\right|$ that were played in the main game, and (ii) for each $j$ and $k$, a game on the trees ${ }_{\star} \mathfrak{T}_{a_{j}}^{a_{j+1}}$ and ${ }_{\star} \mathfrak{S}_{b_{k}}^{b_{k+1}}$ where the nodes played in its earlier rounds are those that have been played in the main game on $\mathfrak{T}^{\prime}$ and $\mathfrak{S}^{\prime}$ and that belong to ${ }_{\star} \mathfrak{T}_{a_{j}}^{a_{j+1}}$ and $\star \mathfrak{S}_{b_{k}}^{b_{k+1}}$.

Let $0 \leqslant i \leqslant n-1$ and suppose that, after round $i$ of the game, the nodes that have been chosen by the two players so far are $t_{1}, \ldots, t_{i} \in$ $\left|\mathfrak{T}^{\prime}\right|$ and $s_{1}, \ldots, s_{i} \in\left|\mathfrak{S}^{\prime}\right|$ (if $i=0$ then the game is yet to begin). Suppose that Player I chooses the node $t_{i+1} \in\left|\mathfrak{T}^{\prime}\right|$ for his $(i+1)$-th turn. We now describe how Player II can choose a response $s_{i+1} \in\left|\mathfrak{S}^{\prime}\right|$ that will allow her to eventually win this game. (The case where, for the $(i+1)$-th round of the game, Player I instead chose a node $s_{i+1} \in\left|\mathfrak{S}^{\prime}\right|$, is handled similarly but with obvious modifications.)

Let $j=t_{i+1}^{\tau}$. Consider for a moment the $n$-round game on $\tau$ and $\sigma$. Note that there are $\tau$ many sets of the form $T_{a_{m}}^{a_{m+1}}$, and $\sigma$ many sets of the form $S_{b_{m}}^{b_{m+1}}$. Suppose that Player II plays this game using the winning strategy she has for it (since $\tau \equiv_{n} \sigma$ ) and that, after round $i$, the elements that have been chosen so far in this game are $t_{1}^{\tau}, \ldots, t_{i}^{\tau} \in \tau$ and $s_{1}^{\sigma}, \ldots, s_{i}^{\sigma} \in \sigma$. Let the $(i+1)$-th move of Player II, in response to Player I's choice of $j \in \tau$ as his $(i+1)$-th move for this game, be the element $k \in \sigma$.

Next consider the $n$-round game on the structures $\star_{\mathfrak{T}_{a_{j}}}^{a_{j+1}}$ and ${ }_{\star} \mathfrak{S}_{b_{k}}^{b_{k+1}}$. Player II has a winning strategy for this game since ${ }_{\star} \mathfrak{T}_{a_{j}}^{a_{j+1}} \equiv_{n}$ ${ }_{\star} \mathfrak{S}_{b_{k}}^{b_{k+1}}$. Let $u_{1}, \ldots, u_{p}$ be all the nodes from amongst $t_{1}, \ldots, t_{i}$ such that $u_{1}, \ldots, u_{p} \in\left|{ }_{\star} \mathfrak{T}_{a_{j}}^{a_{j+1}}\right|$, and let $v_{1}, \ldots, v_{p}$ be all the nodes from amongst $s_{1}, \ldots, s_{i}$ such that $v_{1}, \ldots, v_{p} \in\left|{ }_{\star} \mathfrak{S}_{b_{k}}^{b_{k+1}}\right|$. Suppose that the response of Player II, using her winning strategy for this game, to Player I's choice of the node $t_{i+1} \in\left|{ }_{\star} \mathfrak{T}_{a_{j}}^{a_{j+1}}\right|$ for his $(p+1)$-th move, assuming that the nodes played so far in this game are $u_{1}, \ldots, u_{p} \in$ $\left|{ }_{\star} \mathfrak{T}_{a_{j}}^{a_{j+1}}\right|$ and $v_{1}, \ldots, v_{p} \in\left|{ }_{\star} \mathfrak{S}_{b_{k}}^{b_{k+1}}\right|$, is the node $v_{p+1} \in\left|{ }_{\star} \mathfrak{S}_{b_{k}}^{b_{k+1}}\right|$.

Returning now to the $n$-round game on $\mathfrak{T}^{\prime}$ and $\mathfrak{S}^{\prime}$, Player II's $(i+1)$-th move, in response to Player I having chosen the node $t_{i+1} \in$ $\left|\mathfrak{T}^{\prime}\right|$ for his $(i+1)$-th move, is then the node $s_{i+1}=v_{p+1}$.

At the end of the game, let $\bar{t}=\left(t_{1}, \ldots, t_{n}\right)$ and $\bar{s}=\left(s_{1}, \ldots, s_{n}\right)$. To show that $\left(\mathfrak{T}^{\prime} ; \bar{t}\right)$ and $\left(\mathfrak{S}^{\prime} ; \bar{s}\right)$ satisfy the same atomic sentences (and hence that Player II wins the game, from which it then follows that $\mathfrak{T}^{\prime} \equiv_{n} \mathfrak{S}^{\prime}$ ), it suffices to show, for all $i$ and $j$, that (i) $t_{i}=t_{j}$ iff $s_{i}=s_{j}$, that (ii) $t_{i}<t_{j}$ iff $s_{i}<s_{j}$ and that (iii) $t_{i}<a_{q}$ for some $q$ iff $s_{i}<b_{r}$ for some $r$ (because $\mathfrak{T}^{\prime} \models A\left(t_{i} / x\right)$ iff $t_{i}<a_{q}$ for some $q$, and $\mathfrak{S}^{\prime} \models B\left(s_{i} / x\right)$ iff $s_{i}<b_{r}$ for some $\left.r\right)$. 
It is straightforward to verify that the winning strategy used by Player II above for the game on $\sigma$ and $\tau$, and her winning strategies for the various games on the trees of form ${ }_{\star} \mathfrak{T}_{a_{j}}^{a_{j+1}}$ and ${ }_{\star} \mathfrak{S}_{b_{k}}^{b_{k+1}}$, together with the structure of the trees $\mathfrak{T}^{\prime}$ and $\mathfrak{S}^{\prime}$, guarantee that (i)-(iii) do indeed hold.

Lemma 6 Let $\mathfrak{T}=\left(T ;<_{T}\right)$ and $\mathfrak{S}=\left(S ;<_{S}\right)$ be trees. Consider the following configurations of nodes:

- $\bar{a}=\left(a_{1}, \ldots, a_{k}\right)$, where $a_{1}, \ldots, a_{k} \in T$ with $a_{1}, \ldots, a_{k-1} \in C\left(a_{k}\right)$;

- $\bar{b}=\left(b_{1}, \ldots, b_{k}\right)$, where $b_{1}, \ldots, b_{k} \in S$ with $b_{1}, \ldots, b_{k-1} \in C\left(b_{k}\right)$;

- $\bar{c}=\left(c_{1}, \ldots, c_{m}\right)$, where $c_{1}, \ldots, c_{m} \in T$ with $c_{1}, \ldots, c_{m} \in a_{k}^{\leqslant}$; and

- $\bar{d}=\left(d_{1}, \ldots, d_{m}\right)$, where $d_{1}, \ldots, d_{m} \in S$ with $d_{1}, \ldots, d_{m} \in b_{k}^{\leqslant}$.

If $\left({ }_{\star} \mathfrak{T}^{a_{k}} ; a_{1}, \ldots, a_{k-1}\right) \equiv_{n}\left({ }_{\star} \mathfrak{S}^{b_{k}} ; b_{1}, \ldots, b_{k-1}\right)$ and $\left(\mathfrak{T}_{a_{k}} ; \bar{c}\right) \equiv_{n}\left(\mathfrak{S}_{b_{k}} ; \bar{d}\right)$ then $(\mathfrak{T} ; \bar{a}, \bar{c}) \equiv_{n}(\mathfrak{S} ; \bar{b}, \bar{d})$.

Proof Suppose that $\left({ }_{\star} \mathfrak{T}^{a_{k}} ; a_{1}, \ldots, a_{k-1}\right) \equiv_{n}\left({ }_{\star} \mathfrak{S}^{b_{k}} ; b_{1}, \ldots, b_{k-1}\right)$ and $\left(\mathfrak{T}_{a_{k}} ; \bar{c}\right) \equiv_{n}\left(\mathfrak{S}_{b_{k}} ; \bar{d}\right)$ and let $\sigma$ and $\tau$ be winning strategies for Player II for the $n$-round Ehrenfeucht-Fraïssé games on $\left({ }_{\star} \mathfrak{T}^{a_{k}} ; a_{1}, \ldots, a_{k-1}\right)$ and $\left({ }_{\star} \mathfrak{S}^{b_{k}} ; b_{1}, \ldots, b_{k-1}\right)$, and on $\left(\mathfrak{T}_{a_{k}} ; \bar{c}\right)$ and $\left(\mathfrak{S}_{b_{k}} ; \bar{d}\right)$, respectively. A winning strategy for Player II for the $n$-round game on $(\mathfrak{T} ; \bar{a}, \bar{c})$ and $(\mathfrak{S} ; \bar{b}, \bar{d})$ is obtained by combining her winning strategies $\sigma$ and $\tau$ as follows.

Whenever Player I chooses a node from $T^{a_{k}}$ (respectively $S^{b_{k}}$ ), then, based on all the nodes that have already been chosen from the sets $T^{a_{k}}$ and $S^{b_{k}}$, Player II uses her strategy $\sigma$ to choose a node from $S^{b_{k}}$ (respectively $T^{a_{k}}$ ), and whenever Player I chooses a node from $T_{a_{k}}$ (respectively $S_{b_{k}}$ ), then, based on all the nodes that have already been chosen from the sets $T_{a_{k}}$ and $S_{b_{k}}$, Player II uses her strategy $\tau$ to choose a node from $S_{b_{k}}$ (respectively $T_{a_{k}}$ ).

At the end of the game, let the nodes that were chosen by the two players be $t_{1}, \ldots, t_{n} \in T$ and $s_{1}, \ldots, s_{n} \in S$ and let $\bar{t}=\left(t_{1}, \ldots, t_{n}\right)$ and $\bar{s}=\left(s_{1}, \ldots, s_{n}\right)$. To confirm that the structures $(\mathfrak{T} ; \bar{a}, \bar{c}, \bar{t})$ and $(\mathfrak{S} ; \bar{b}, \bar{d}, \bar{s})$ satisfy the same atomic formulas (and hence that Player II wins the game), it suffices to check the following for all $i$ and $j$ : (i) $a_{i}=a_{j} \Leftrightarrow b_{i}=b_{j}$, (ii) $a_{i}<a_{j} \Leftrightarrow b_{i}<b_{j}$, (iii) $c_{i}=c_{j} \Leftrightarrow d_{i}=d_{j}$, (iv) $c_{i}<c_{j} \Leftrightarrow d_{i}<d_{j}$, (v) $a_{i}=c_{j} \Leftrightarrow b_{i}=d_{j}$, (vi) $a_{i}<c_{j} \Leftrightarrow b_{i}<d_{j}$, (vii) $c_{i}<a_{j} \Leftrightarrow d_{i}<b_{j}$, (viii) $t_{i}=t_{j} \Leftrightarrow s_{i}=s_{j}$, (ix) $t_{i}<t_{j} \Leftrightarrow s_{i}<s_{j}$, (x) $t_{i}=a_{j} \Leftrightarrow s_{i}=b_{j}$, (xi) $t_{i}<a_{j} \Leftrightarrow s_{i}<b_{j}$, (xii) $a_{i}<t_{j} \Leftrightarrow b_{i}<s_{j}$, (xiii) $t_{i}=c_{j} \Leftrightarrow s_{i}=d_{j}$, (xiv) $t_{i}<c_{j} \Leftrightarrow s_{i}<d_{j}$, and, finally, (xv) $c_{i}<t_{j} \Leftrightarrow d_{i}<s_{j}$.

It is straightforward to check that each of (i)-(xv) does indeed hold. It hence follows that $(\mathfrak{T} ; \bar{a}, \bar{c}) \equiv_{n}(\mathfrak{S} ; \bar{b}, \bar{d})$. 


\section{Canonical form of formulas that de- fine paths}

Lemma 7 (Elimination of parameters) Let $\mathfrak{T}$ be any tree. Let $\bar{a}=\left(a_{1}, \ldots, a_{k}\right)$ be a $k$-tuple of nodes from $T$ such that $a_{1}, \ldots, a_{k-1} \in$ $C\left(a_{k}\right)$ and let $b, c \in a_{k}^{\leqslant}$. Let $n$ be a natural number. The following statements are equivalent:

(i) $(\mathfrak{T} ; b, \bar{a}) \equiv_{n}(\mathfrak{T} ; c, \bar{a})$

(ii) $\left(\mathfrak{T} ; b, a_{k}\right) \equiv_{n}\left(\mathfrak{T} ; c, a_{k}\right)$

(iii) $\left(\mathfrak{T}_{a_{k}} ; b\right) \equiv_{n}\left(\mathfrak{T}_{a_{k}} ; c\right)$

Proof (i) $\Rightarrow$ (ii): Immediate.

(ii) $\Rightarrow$ (iii): Suppose that $\left(\mathfrak{T} ; b, a_{k}\right) \equiv_{n}\left(\mathfrak{T} ; c, a_{k}\right)$. Then Player II has a winning strategy for the $n$-round Ehrenfeucht-Fraïssé game on the trees $\left(\mathfrak{T} ; b, a_{k}\right)$ and $\left(\mathfrak{T} ; c, a_{k}\right)$. Clearly this winning strategy will require that, whenever Player I chooses a node from $a_{k}^{\leqslant}$in either of these two trees then Player II responds with a node from $a_{k}^{\leqslant}$in the other tree. It follows that Player II has a winning strategy for the $n$-round game on the trees $\left(\mathfrak{T}_{a_{k}} ; b\right)$ and $\left(\mathfrak{T}_{a_{k}} ; c\right)$ hence $\left(\mathfrak{T}_{a_{k}} ; b\right) \equiv_{n}$ $\left(\mathfrak{T}_{a_{k}} ; c\right)$, as required.

(iii) $\Rightarrow(\mathrm{i})$ : Suppose that $\left(\mathfrak{T}_{a_{k}} ; b\right) \equiv_{n}\left(\mathfrak{T}_{a_{k}} ; c\right)$. Since

$$
\left({ }_{\star} \mathfrak{T}^{a_{k}} ; a_{1}, \ldots, a_{k-1}\right) \equiv_{n}\left({ }_{\star} \mathfrak{T}^{a_{k}} ; a_{1}, \ldots, a_{k-1}\right)
$$

then by Lemma $6,(\mathfrak{T} ; b, \bar{a}) \equiv_{n}(\mathfrak{T} ; c, \bar{a})$.

Corollary 8 Let $\mathfrak{T}$ be any tree. Let $\bar{a}=\left(a_{1}, \ldots, a_{k}\right)$ be a $k$-tuple of nodes from $T$ such that $a_{1}, \ldots, a_{k-1} \in C\left(a_{k}\right)$ and let $b, c \in a_{k}^{\leqslant}$. Let $n$ be a natural number. The following statements are equivalent:

(i) $(\mathfrak{T} ; \bar{a}) \models \llbracket(\mathfrak{T} ; b, \bar{a}) \rrbracket^{n}(c / x, \bar{a})$

(ii) $\left(\mathfrak{T} ; a_{k}\right) \models \llbracket\left(\mathfrak{T} ; b, a_{k}\right) \rrbracket^{n}\left(c / x, a_{k}\right)$

(iii) $\mathfrak{T}_{a_{k}} \models \llbracket\left(\mathfrak{T}_{a_{k}} ; b\right) \rrbracket^{n}(c / x)$

Proof By Theorem 3 and Lemma 7.

Every path that contains a leaf can be defined using at most one parameter chosen from the path itself: If $A$ is a path in a tree $\mathfrak{T}$ and $A$ has a leaf $a$ then $A$ can be defined in $(\mathfrak{T} ; a)$ by the formula $\varphi(x, a)=x \leqslant a$. The next theorem shows that every leafless path that is at all definable can be defined using at most one parameter chosen from the path itself. 
Theorem 9 (Canonical form of path-defining formulas) Let $\mathfrak{T}$ be any tree. Let $\bar{a}=\left(a_{1}, \ldots, a_{k}\right)$ be a tuple of nodes from $T$ and let $A$ be a leafless path that is defined in $(\mathfrak{T} ; \bar{a})$ by a formula $\varphi(x, \bar{a})$ of quantifier rank $m$. For every $b \in A$ such that $a_{1}, \ldots, a_{k} \in C(b) \cup\{b\}$, there exists an $m$-characteristic formula $\tau(x, y)$ such that $A$ is defined in $(\mathfrak{T} ; b)$ by the formula

$$
\psi(x, b)=\exists z(x<z \wedge \tau(z, b)) .
$$

Proof Let $b \in A$ with $a_{1}, \ldots, a_{k} \in C(b) \cup\{b\}$ and note that $A$ is defined in $(\mathfrak{T} ; \bar{a} b)$ by the formula $\varphi^{\prime}(x, \bar{a} b)=\varphi(x, \bar{a})$. Let the cofinality of the stem $A \cap b^{\leqslant}$be $\alpha$ and let $\left(b_{i}\right)_{i \in \alpha}$ be a sequence that is cofinal in the stem $A \cap b^{\leqslant}$. Since there are only finitely many pairwise nonequivalent $m$-characteristic formulas over 2-tuples in the language of trees, it follows from the infinite pigeonhole principle there must exist an $m$-characteristic formula $\tau(x, y)$ and a subsequence $\left(c_{i}\right)_{i \in \alpha}$ of $\left(b_{i}\right)_{i \in \alpha}$ such that $(\mathfrak{T} ; b) \models \tau\left(c_{i} / x, b\right)$ for every $i \in \alpha$, and the sequence $\left(c_{i}\right)_{i \in \alpha}$ will also be cofinal in the stem $A \cap b^{\leqslant}$. Since, in particular, $(\mathfrak{T} ; b) \models \tau\left(c_{0} / x, b\right)$, then by Theorem $3, \tau(x, y) \equiv \llbracket\left(\mathfrak{T} ; c_{0}, b\right) \rrbracket^{m}(x, y)$ hence $(\mathfrak{T} ; b) \models \llbracket\left(\mathfrak{T} ; c_{0}, b\right) \rrbracket^{m}\left(c_{i} / x, b\right)$ for each $i \in \alpha$. By Corollary 8 it follows that

$$
\begin{aligned}
(\mathfrak{T} ; \bar{a} b) \models \llbracket\left(\mathfrak{T} ; c_{0}, \bar{a} b\right) \rrbracket^{m}(u / x, \bar{a} b) \Leftrightarrow \\
(\mathfrak{T} ; b) \models \llbracket\left(\mathfrak{T} ; c_{0}, b\right) \rrbracket^{m}(u / x, b)
\end{aligned}
$$

for every $u \in b^{\leqslant}$.

For every $u \in b^{\leqslant} \backslash A,(\mathfrak{T} ; \bar{a} b) \not \models \llbracket\left(\mathfrak{T} ; c_{0}, \bar{a} b\right) \rrbracket^{m}(u / x, \bar{a} b)$ : If $(\mathfrak{T} ; \bar{a} b) \models$ $\llbracket\left(\mathfrak{T} ; c_{0}, \bar{a} b\right) \rrbracket^{m}(u / x, \bar{a} b)$ then by Theorem $3,(\mathfrak{T} ; u, \bar{a} b) \equiv_{m}\left(\mathfrak{T} ; c_{0}, \bar{a} b\right)$ and since $(\mathfrak{T} ; \bar{a} b) \models \varphi^{\prime}\left(c_{0} / x, \bar{a} b\right)$ then $(\mathfrak{T} ; \bar{a} b) \models \varphi^{\prime}(u / x, \bar{a} b)$, a contradiction with the fact that $\varphi^{\prime}(x, \bar{a} b)$ defines $A$ in $(\mathfrak{T} ; \bar{a} b)$.

Hence by $(3),(\mathfrak{T} ; b) \not \models \llbracket\left(\mathfrak{T} ; c_{0}, b\right) \rrbracket^{m}(u / x, b)$ for every $u \in b^{\leqslant} \backslash A$.

Moreover, $(\mathfrak{T} ; b) \not \models \llbracket\left(\mathfrak{T} ; c_{0}, b\right) \rrbracket^{m}(u / x, b)$ for every $u \in C(b)$, because $(\mathfrak{T} ; b) \models \llbracket\left(\mathfrak{T} ; c_{0}, b\right) \rrbracket^{m}(u / x, b)$ only if $b \leqslant u$.

Since, however, $(\mathfrak{T} ; b) \models \llbracket\left(\mathfrak{T} ; c_{0}, b\right) \rrbracket^{m}\left(c_{i} / x, b\right)$ for each $i \in \alpha$, and since $A$ consists precisely of those nodes that lie below a node $c_{i}$ for some $i \in \alpha$, it follows that $A$ can be defined in $(\mathfrak{T} ; b)$ using the formula

$$
\psi(x, b)=\exists z\left(x<z \wedge \llbracket\left(\mathfrak{T} ; c_{0}, b\right) \rrbracket^{m}(z, b)\right),
$$

as required.

Obviously the formula $\psi(x, b)$ given in the above theorem is not the formula of lowest quantifier rank to define $A$ (since $\operatorname{qr}(\psi)=\operatorname{qr}(\varphi)+1$ where $\varphi(x, \bar{a})$ is known to define $A)$. Furthermore, it may actually be the case that $A$ can be defined without any parameters whatsoever. 
Corollary 10 Let $\mathfrak{T}$ be any tree. For every node $a \in T$ and for every natural number $n$, there are only finitely many paths in $\mathfrak{T}$ that pass through $a$ and can be defined by a formula $\varphi(x, a)$ of quantifier rank $n$ in the tree $(\mathfrak{T} ; a)$.

Proof By Theorem 9 and the fact that there are only finitely many pairwise non-equivalent characteristic formulas of a given quantifier rank over tuples of a given length.

\section{Trees that have only finitely many undefinable paths}

Proposition 11 Let $\mathfrak{T}$ be any tree, let $A$ be a path in $\mathfrak{T}$, let $a \in A$ and let $B=A \cap a^{\leqslant}$. Then $A$ can be defined by a formula in $(\mathfrak{T} ; a)$ if and only if $B$ can be defined by a formula in $\mathfrak{T}_{a}$.

Proof If $A$ has a leaf then the result is trivial, so consider the case where $A$ does not have a leaf. First suppose that $A$ can be defined by a formula in $(\mathfrak{T} ; a)$. By Theorem 9 there exists a node $c \in B(c$ is the node $c_{0}$ in the proof of Theorem 9) and natural number $k$ such that the formula $\varphi(x, a)=\exists y\left(x<y \wedge \llbracket(\mathfrak{T} ; c, a) \rrbracket^{k}(y, a)\right)$ defines $A$ in $(\mathfrak{T} ; a)$. By Corollary 8 ,

$$
(\mathfrak{T} ; a) \models \llbracket(\mathfrak{T} ; c, a) \rrbracket^{k}(u / x, a) \Leftrightarrow \mathfrak{T}_{a} \models \llbracket\left(\mathfrak{T}_{a} ; c\right) \rrbracket^{k}(u / x)
$$

for each $u \in a^{\leqslant}$. It follows that $B$ is defined in $\mathfrak{T}_{a}$ by the formula $\psi(x)=\exists y\left(x<y \wedge \llbracket\left(\mathfrak{T}_{a} ; c\right) \rrbracket^{k}(y)\right)$.

Conversely, suppose that $\varphi(x)$ is any formula that defines the path $B$ in $\mathfrak{T}_{a}$. Since $\mathfrak{T}_{a}=(\mathfrak{T} ; a)^{a \leqslant x}$ then by Proposition 1 , the formula $\varphi^{a \leqslant x}(x, a)$ defines the set $B$ in $(\mathfrak{T} ; a)$. Hence the formula $\psi(x, a)=$ $x<a \vee \varphi^{a \leqslant x}(x, a)$ defines the path $A$ in $(\mathfrak{T} ; a)$.

Proposition 12 Let $\mathfrak{T}$ be any tree and let $a \in T$. Let $A$ be a path in $\mathfrak{T}$ that does not contain a (i.e. $A \subseteq C(a)$ ). Then $A$ is definable in $\mathfrak{T}$ if and only if $A$ is definable in $\mathfrak{T}^{a}$.

Proof If $A$ has a leaf $c$ then the formula $x \leqslant c$ defines $A$ in both of the trees $(\mathfrak{T} ; c)$ and $\left(\mathfrak{T}^{a} ; c\right)$. Hence consider the case where $A$ does not have a leaf.

First suppose that the path $A$ is definable in $\mathfrak{T}$. By Theorem 9 it follows that there exists $b \in A$ with $a \in C(b)$ (hence $b \nless a$ ) such that $A$ can be defined by a formula in $(\mathfrak{T} ; b)$. By Proposition $11, A \cap b^{\leqslant}$can 
be defined by a formula in $\mathfrak{T}_{b}$ so by another application of Proposition 11 in the context of the tree $\mathfrak{T}^{a}\left(\right.$ since $\left.\left|\mathfrak{T}_{b}\right| \subseteq\left|\mathfrak{T}^{a}\right|\right), A$ can be defined by a formula in $\left(\mathfrak{T}^{a} ; b\right)$.

Next suppose that $A$ is definable in $\mathfrak{T}^{a}$. By Theorem 9 there exists $b \in A$ and a formula $\varphi(x, b)$ that defines $A$ in $\left(\mathfrak{T}^{a} ; b\right)$. Since $\left(\mathfrak{T}^{a} ; b\right)=$ $(\mathfrak{T} ; b, a)^{\neg(a \leqslant x)}$ then by Proposition 1 it follows that $\varphi^{\neg(a \leqslant x)}(x, b, a)$ defines $A$ in $(\mathfrak{T} ; b, a)$.

Proposition 13 Let $\mathfrak{T}$ be any tree and let $A$ be a path that is not definable in $\mathfrak{T}$. For every $a \in A$ and for every natural number $n$, there exists $b \in a^{\leqslant} \cap A$ and $c \in a^{\leqslant} \backslash A$ such that $\mathfrak{T}_{b} \equiv_{n} \mathfrak{T}_{c}$.

Proof Let $a \in A$ and let $n$ be a natural number, but suppose to the contrary that $\mathfrak{T}_{u} \neq_{n} \mathfrak{T}_{v}$ for every $u \in a^{\leqslant} \cap A$ and $v \in a^{\leqslant} \backslash A$. Let $\tau_{1}, \ldots, \tau_{m}$ be all the $n$-characteristic sentences in the language of trees, up to logical equivalence. Let $I=\left\{i: \mathfrak{T}_{u} \models \tau_{i}\right.$ for some $\left.u \in a^{\leqslant} \cap A\right\}$. Then for every $u \in a^{\leqslant}$we have that $\mathfrak{T}_{u} \models \tau_{i}$ for some $i \in I$ if and only if $u \in A$. But then $A$ can be defined in (T; $a)$ using the formula

$$
\varphi(x, a)=x<a \vee a \leqslant x \wedge\left(\bigvee_{i \in I} \tau_{i}^{\theta(w, x)}(x)\right)
$$

where $\theta(w, x)=x \leqslant w$, a contradiction.

Proposition 14 Let $\mathfrak{T}$ be a tree that has only finitely many paths that are not definable. For every natural number $n$, there exists a tree $\mathfrak{S}$ of which every path is definable, and such that $\mathfrak{S} \equiv_{n} \mathfrak{T}$.

Proof Let $n$ be a natural number. Let $A$ be a path in $\mathfrak{T}$ that is not definable. Since $\mathfrak{T}$ has only finitely many paths that are not definable then there exists $a \in A$ such that $A$ is the only path in $\mathfrak{T}$ that contains $a$ and is not definable. By Proposition 13 there exists $b \in a^{\leqslant} \cap A$ and $c \in a^{\leqslant} \backslash A$ such that $\mathfrak{T}_{b} \equiv_{n} \mathfrak{T}_{c}$. Let $\mathfrak{T}^{\prime}$ be the tree that is obtained from $\mathfrak{T}$ by replacing $\mathfrak{T}_{b}$ with $\mathfrak{T}_{c}$. Since ${ }_{\star} \mathfrak{T}^{b} \equiv_{n}{ }_{\star} \mathfrak{T}^{b}$ then it follows by Lemma 6 that $\mathfrak{T}^{\prime} \equiv_{n} \mathfrak{T}$.

Every path in $\mathfrak{T}$ that contains $c$ is definable hence by Proposition 11, every path in $\mathfrak{T}_{c}$ is definable. By another application of Proposition 11 , every path in $\mathfrak{T}^{\prime}$ that contains the node $c$ from the copy of $\mathfrak{T}_{c}$ that was substituted for $\mathfrak{T}_{b}$, is definable in $\mathfrak{T}^{\prime}$.

Moreover, by Proposition 12, every path $X$ in $\mathfrak{T}$ for which $X \subseteq$ $C(b)$ that is definable in $\mathfrak{T}$, is definable in $\mathfrak{T}^{b}$ as well, so by a second application of Proposition 12 in the context of the tree $\mathfrak{T}^{\prime}$, such a path $X$ is also definable in $\mathfrak{T}^{\prime}$. 
Hence the tree $\mathfrak{T}^{\prime}$ has one fewer path that is not definable than the tree $\mathfrak{T}$ : every path in $\mathfrak{T}^{\prime}$ that contains the node $c$ from the copy of $\mathfrak{T}_{c}$ that was substituted for $\mathfrak{T}_{b}$, is definable in $\mathfrak{T}^{\prime}$, while every path in the tree $\mathfrak{T}^{\prime}$ that is contained in $C(b)$ and is a definable path in $\mathfrak{T}$, is also definable in $\mathfrak{T}^{\prime}$.

Repeat this construction for every other path in $\mathfrak{T}$ that is not definable so as to eventually obtain a tree $\mathfrak{S}$ with the property that every path in $\mathfrak{S}$ is definable and $\mathfrak{S} \equiv_{n} \mathfrak{T}$.

\section{The partition and cover properties}

\subsection{The partition property}

Let $\mathfrak{L}=(L ;<)$ be a linear order and let $C$ be a finite set. A function $f:\left\{(x, y) \in L^{2}: x<y\right\} \rightarrow C$ is called a colouring of $\mathfrak{L}$. The colouring $f$ is called additive when the following condition holds: for all $x_{1}, y_{1}, z_{1} \in L$ and $x_{2}, y_{2}, z_{2} \in L$ with $x_{1}<y_{1}<z_{1}$ and $x_{2}<y_{2}<z_{2}$, if $f\left(\left(x_{1}, y_{1}\right)\right)=f\left(\left(x_{2}, y_{2}\right)\right)$ and $f\left(\left(y_{1}, z_{1}\right)\right)=f\left(\left(y_{2}, z_{2}\right)\right)$ then $f\left(\left(x_{1}, z_{1}\right)\right)=f\left(\left(x_{2}, z_{2}\right)\right)$. An increasing sequence $\left(a_{i}\right)_{i \in \alpha}$ in $L$ is called homogeneous in $\mathfrak{L}$ if there is an element $c \in C$ such that $f\left(\left(a_{i}, a_{j}\right)\right)=c$ for all $i<j$. The following version of Ramsey's Theorem is proved in [14].

Theorem 15 ([14]) If $f$ is an additive colouring of a limit ordinal $\alpha$ then there exists a sequence $\left(x_{i}\right)_{i \in \beta}$ that is cofinal and homogeneous in $\alpha$.

Now consider any tree $\mathfrak{T}$. Let $A$ be a leafless path of cofinality $\alpha$ in $\mathfrak{T}$ ( $\alpha$ will be a limit ordinal since $A$ is leafless) and let $\left(b_{i}\right)_{i \in \alpha}$ be a sequence that is cofinal in $A$. Consider the set $C=\left\{\llbracket \star_{\star} \mathfrak{T}_{b_{i}}^{b_{j}} \rrbracket^{n}: i<j\right\}$ and let $C / \equiv$ be the quotient set of $C$ under the equivalence relation of logical equivalence. The set $C / \equiv$ will be finite.

Define the function $f:\{(i, j) \in \alpha \times \alpha: i<j\} \rightarrow C / \equiv$ by specifying that $\left.f((i, j))=\left[\llbracket \star^{*} \mathfrak{T}_{b_{i}}^{b_{j}}\right]^{n}\right]_{\equiv}$, where $[\tau]_{\equiv}$ denotes the equivalence class in $C / \equiv$ that contains $\tau$. The function $f$ is a colouring of $\alpha$, and from Theorem 3 and Lemma 4 it follows that $f$ is additive. Hence by Theorem 15, together with the fact that $\alpha$ is a regular ordinal, there exists a sequence $\left(x_{i}\right)_{i \in \alpha}$ that is cofinal and homogeneous in $\alpha$ under $f$. Let $a_{i}=b_{x_{i}}$ for every $i \in \alpha$. Then for $p, q, s, t \in \alpha$ with $p<q$ and $s<t$, the homogeneity of $\left(x_{i}\right)_{i \in \alpha}$ together with Theorem 3 gives that ${ }_{\star} \mathfrak{T}_{a_{p}}^{a_{q}} \equiv_{n \star} \mathfrak{T}_{a_{s}}^{a_{t}}$.

We have hence obtained the following result for trees. 
Partition Property: Let $\mathfrak{T}$ be any tree and let $A$ be a leafless path in $\mathfrak{T}$. For every natural number $n$, there is an ordinal $\alpha$ and a sequence $\left(a_{i}\right)_{i \in \alpha}$ that is cofinal in $A$ such that $\mathfrak{T}_{a_{p}}^{a_{q}} \equiv_{n \star} \mathfrak{T}_{a_{s}}^{a_{t}}$ for all $p<q$ and $s<t$.

\subsection{The cover property}

Let $A$ be a stem (hence possibly also a path) with no greatest node in a tree $\mathfrak{T}$. A sequence $\left(a_{i}\right)_{i \in \alpha}$ that is cofinal in $A$ will be called a cover for $(\mathfrak{T} ; A)$. If $\left(a_{i}\right)_{i \in \alpha}$ is a cover for $(\mathfrak{T} ; A)$ then, since $A$ has no greatest node, $\alpha$ must be a limit ordinal, $A=\bigcup_{i \in \alpha} a_{i}^{>}$and $\star^{A}=\bigcup_{i \in \alpha} \star \mathfrak{T}^{a_{i}}$.

Consider the following cover property of trees:

$\mathrm{CP}$ : Let $n$ be any natural number. Let $\left(a_{i}\right)_{i \in \alpha}$ and $\left(b_{i}\right)_{i \in \alpha}$ be covers for the trees $(\mathfrak{T} ; A)$ and $(\mathfrak{S} ; B)$ respectively. If $\left(\mathfrak{T} ; a_{i}\right) \equiv_{n}\left(\mathfrak{S} ; b_{i}\right)$ for every $i$ then ${ }_{\star} \mathfrak{T}^{A} \equiv_{n \star} \mathfrak{S}^{B}$.

The above cover property is false, as will be shown in Example 16. We first describe a method that will be used in Example 16 for constructing trees.

A tree $\mathfrak{T}$ is called well-founded when every non-empty set of nodes in $T$ has a minimal element. The height of a node $a \in T$ for wellfounded $\mathfrak{T}$ is the order type (chosen from the class of ordinals) $h(a)$ of the set $a^{>}$. The height of $\mathfrak{T}$ is the ordinal $\bigcup_{a \in T}(h(a)+1)$.

Let $A$ be a non-empty set and let $\alpha$ be an ordinal. For each ordinal $\beta$ with $\beta<\alpha$, define the set $G_{\beta}$ recursively as follows:

- (Initial value.) Let $G_{0}:=\{()\}$. The empty sequence () represents the root of the tree.

- (To find $G_{\beta+1}$ given $G_{\beta}$.) Let $\beta$ be any ordinal with $\beta<\alpha$ and let the set $G_{\beta}$ be known. Choose any function $f_{\beta+1}: G_{\beta} \rightarrow \mathcal{P}(A)$ such that $f_{\beta+1}(\bar{x}) \neq \emptyset$ for at least one tuple $\bar{x} \in G_{\beta}$. Then

$$
G_{\beta+1}:=\left\{\bar{x} y: \bar{x} \in G_{\beta} \text { and } f_{\beta+1}(\bar{x}) \neq \emptyset \text { and } y \in f_{\beta+1}(\bar{x})\right\} .
$$

- (To find $G_{\beta}$ when $\beta$ is a limit ordinal.) Let $\beta$ be a limit ordinal with $\beta<\alpha$ and let the set $G_{\gamma}$ be known for each $\gamma<\beta$. Let

$$
\begin{aligned}
L_{\beta}:=\{\bar{x}: \ell(\bar{x})=\beta \text { and if } \bar{y} \text { is a proper initial sub- } \\
\text { sequence of } \left.\bar{x} \text { then } \bar{y} \in G_{\gamma} \text { for some } \gamma<\beta\right\} .
\end{aligned}
$$

Choose any function $f_{\beta}: L_{\beta} \rightarrow \mathcal{P}(A)$ such that $f_{\beta}(\bar{x}) \neq \emptyset$ for at least one tuple $\bar{x} \in L_{\beta}$. Then

$$
G_{\beta}:=\left\{\bar{x} y: \bar{x} \in L_{\beta} \text { and } f_{\beta}(\bar{x}) \neq \emptyset \text { and } y \in f_{\beta}(\bar{x})\right\} .
$$


Now define $G:=\bigcup_{\beta<\alpha} G_{\beta}$ and $f:=\bigcup_{\beta<\alpha} f_{\beta}$.

For $\bar{x}, \bar{y} \in G$, define $\bar{x}<\bar{y}$ if and only if $\bar{x}$ is a proper initial subsequence of $\bar{y}$. The structure $(G ;<)$ is a well-founded tree of height $\alpha$ and will be denoted as $\mathfrak{G}(\alpha, A, f)$.

We now give the earlier promised counterexample to the property CP.

Example 16 Fix a natural number $n$ with $n \geqslant 6$. We will construct a tree $\mathfrak{T}$, paths $A$ and $B$ in $\mathfrak{T}$, and covers $\left(a_{i}\right)_{i \in \omega}$ and $\left(b_{i}\right)_{i \in \omega}$ for $(\mathfrak{T} ; A)$ and $(\mathfrak{T} ; B)$ respectively, such that $\left(\mathfrak{T} ; a_{i}\right) \equiv_{n}\left(\mathfrak{T} ; b_{i}\right)$ for every $i$ but $\star_{\mathfrak{T}^{A}} \not_{n} \star_{\mathfrak{T}^{B}}$. To show that $\star_{\mathfrak{T}^{A}} \not_{n} \star_{\mathfrak{T}^{B}}$, we will find a sentence $\psi$ of quantifier rank at most 6 such that ${ }_{\star} \mathfrak{T}^{A} \models \psi$ but ${ }_{\star} \mathfrak{T}^{B} \not \models \psi$.

Define the function $h: \mathbb{N} \rightarrow \mathbb{N}$ as follows:

$$
h(i)=2\left(2^{n}+i-1\right)+1 .
$$

Consider the tree $\mathfrak{T}=\mathfrak{G}(\omega,\{0,1,2\}, f)$ with $f=\bigcup_{\beta<\omega} f_{\beta}$ defined recursively as follows:

1. Take $G_{0}=\{()\}$.

2. Let $\beta$ be an ordinal with $\beta<\omega$ and let the set $G_{\beta}$ be given. Define $f_{\beta+1}: G_{\beta} \rightarrow \mathcal{P}(\{0,1,2\})$ as follows:

(a) $f_{1}(())=\{0,1,2\}$.

(b) If $\bar{x}$ has the form $\bar{x}=\bar{y} \overline{0}_{2^{n}}$ where $\ell(\bar{y})=k \cdot 2^{n}$ for some odd natural number $k$, then $f_{\beta+1}(\bar{x})=\{0,1,2\}$.

(c) If the last entry of $\bar{x}$ is 2 then $f_{\beta+1}(\bar{x})=\emptyset$.

(d) Else $f_{\beta+1}(\bar{x})=\{0,1\}$.

In other words, $\mathfrak{T}$ is obtained by taking the binary tree of height $\omega$ and adding one additional successor to the root node and to each node of the form $\bar{y} \overline{0}_{2^{n}}$ with $\ell(\bar{y})=k \cdot 2^{n}$ for some odd natural number $k$.

Let $a_{i}=\overline{0}_{h(i) \cdot 2^{n}}$ and $b_{i}=\overline{0}_{(h(0)-1) \cdot 2^{n}} \overline{1}_{(2 i+1) \cdot 2^{n}}$ for every natural number $i$ and let $A=\bigcup_{i \in \mathbb{N}} a_{i}^{>}$and $B=\bigcup_{i \in \mathbb{N}} b_{i}^{>}$. The paths $A$ and $B$ are depicted in Figure 1.

Then $\left(\mathfrak{T} ; a_{i}\right) \equiv_{n}\left(\mathfrak{T} ; b_{i}\right)$ for each $i$ but ${ }_{\star} \mathfrak{T}^{A}=(\mathfrak{T} ; A) \not \equiv_{n}(\mathfrak{T} ; B)={ }_{\star} \mathfrak{T}^{B}$.

To see that $\left(\mathfrak{T} ; a_{i}\right) \equiv_{n}\left(\mathfrak{T} ; b_{i}\right)$ for each $i$, we show that

$$
\begin{aligned}
& \star \mathbb{T}^{\overline{0}_{(h(i)-1) \cdot 2^{n}}} \equiv_{n} \quad \star T^{\overline{0}_{(h(0)-1) \cdot 2^{n}}},
\end{aligned}
$$

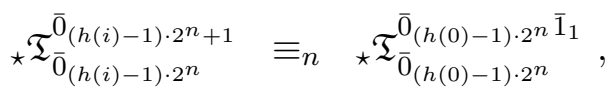

$$
\begin{aligned}
& { }^{T^{T}} \overline{0}_{(h(i)-1) \cdot 2^{n}+1}^{a_{i}} \quad \equiv_{n} \quad \star T_{\overline{0}_{(h(0)-1) \cdot 2^{n}} \overline{1}_{1}}^{b_{i}} \quad \text { and }
\end{aligned}
$$



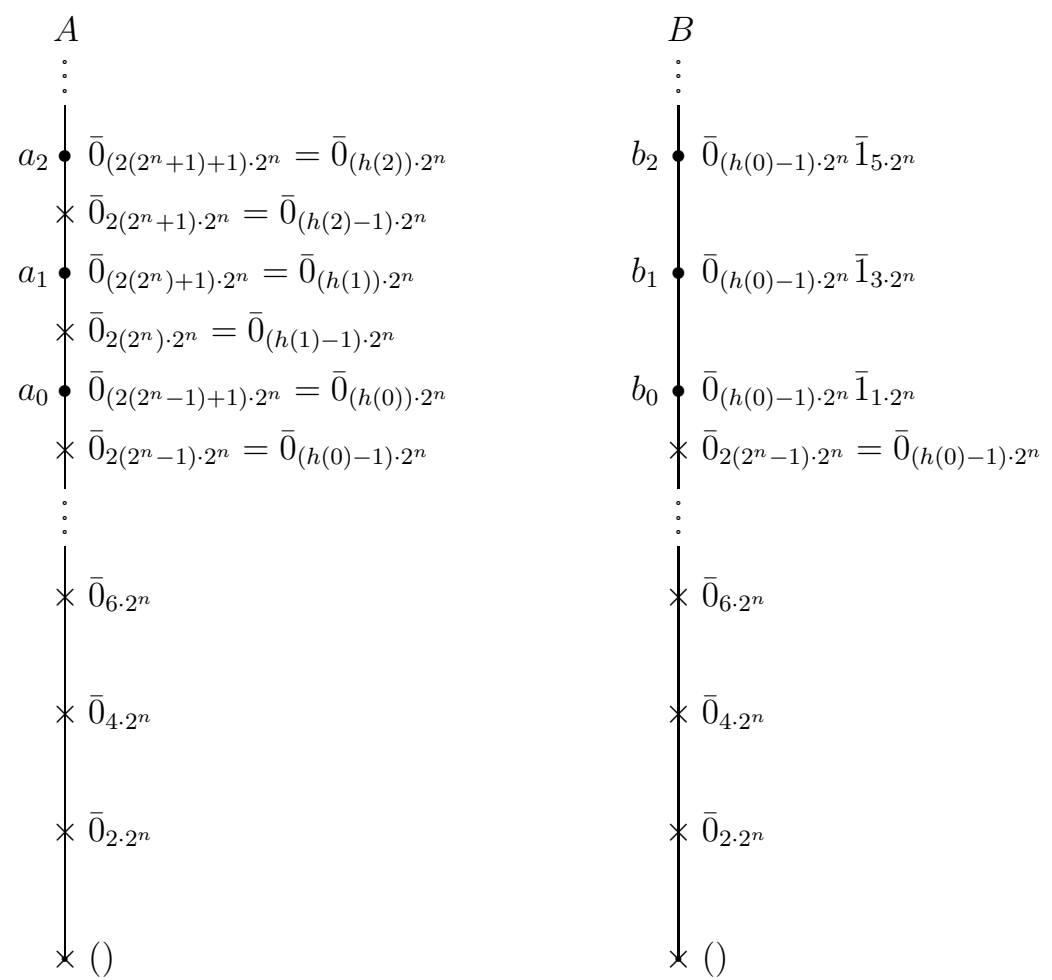

Figure 1: The paths $A$ (left) and $B$ (right) in the tree $\mathfrak{T}$ (see Example 16). Nodes that have three immediate successors are indicated with the symbol $\times$.

$$
\mathfrak{T}_{a_{i}} \equiv_{n} \quad \mathfrak{T}_{b_{i}} .
$$

Player II will then have a winning strategy for the n-round Ehrenfeucht-Fraïssé game on the trees $\left(\mathfrak{T} ; a_{i}\right)$ and $\left(\mathfrak{T} ; b_{i}\right)$ by combining her winning strategies for the $n$-round games on the four sets of trees given in (4) - (7).

Proof of (4): Note that the greatest node in the stem $\left(\overline{0}_{(h(i)-1) \cdot 2^{n}}\right)^{>}$that has three immediate successors is the node $\overline{0}_{(h(i)-3) \cdot 2^{n}}$ and $h(i)-3=$ $2\left(2^{n}+i-2\right)$, and the greatest node in the stem $\left(\overline{0}_{(h(0)-1) \cdot 2^{n}}\right)^{>}$that has three immediate successors is the node $\overline{0}_{(h(0)-3) \cdot 2^{n}}$ and $h(0)-3=$ $2\left(2^{n}-2\right)$. Now

$$
\star \widetilde{T}_{\overline{0}_{2 p \cdot 2^{n}}}^{\overline{0}_{(2 p+2) \cdot 2^{n}}} \cong \star \mathfrak{T}_{\overline{0}_{2 q \cdot 2^{n}}}^{\overline{0}_{(2 q+2) \cdot 2^{n}}}
$$

for all integers $p$ and $q$ with $0 \leqslant p \leqslant 2^{n}+i-2$ and $0 \leqslant q \leqslant 2^{n}-2$ hence

$$
\star \mathfrak{T}_{\overline{0}_{2 p \cdot 2^{n}}}^{\overline{0}_{(2 p+2) \cdot 2^{n}}} \equiv_{n} \star \mathfrak{T}_{\overline{0}_{2 q \cdot 2^{n}}}^{\overline{0}_{(2 q+2) \cdot 2^{n}}}
$$


for all such $p$ and $q$.

Let $C$ be the set that consists of all nodes $t$ that belong to the stem $\left(\overline{0}_{(h(i)-1) \cdot 2^{n}}\right)^{>} \subseteq A$ such that $t$ has three immediate successors, and let $D$ be the set that consists of all nodes $s$ that belong to the stem $\left(\overline{0}_{(h(0)-1) \cdot 2^{n}}\right)^{>} \subseteq B$ such that $s$ has three immediate successors. Since $|C|,|D| \geqslant 2^{n}-1$ then by (2),

$$
\left(C ;<\uparrow_{C}\right) \equiv_{n}\left(D ;<\uparrow_{D}\right) .
$$

Since there are $|C|$ many trees of the form ${ }_{\star}{\mathfrak{T}^{0}}_{\overline{0}_{2 p \cdot 2^{n}}}^{\bar{T}_{2 p+2) \cdot 2^{n}}}$, and $|D|$ many trees of the form ${ }_{\star} \mathcal{T}_{\overline{0}_{2 q \cdot 2^{n}}}^{\overline{0}_{(2 q+2) \cdot 2^{n}}}$, then it follows from Lemma 5 that

$$
\star \mathbb{T}^{\overline{0}_{(h(i)-1) \cdot 2^{n}}} \equiv_{n} \star \mathfrak{T}^{\overline{0}_{(h(0)-1) \cdot 2^{n}}} .
$$

Proof of (5): This follows (see Figure 2) from the fact that

$$
\star \mathfrak{T}_{\overline{0}_{(h(i)-1) \cdot 2^{n}}}^{\overline{0}_{(h(i)-1) \cdot 2^{n}+1}} \cong \star \mathfrak{T}_{\overline{0}_{(h(0)-1) \cdot 2^{n}}}^{\overline{0}_{(h(0)-1) \cdot 2^{n}} \overline{1}_{1}} .
$$

Proof of (6): Note that

$$
\star \mathfrak{T}_{\overline{0}_{p}}^{\overline{0}_{p+1}} \cong \star_{\mathfrak{T}^{\prime}}^{\overline{0}_{(h(0)-1) \cdot 2^{n}} \overline{1}_{q+1}}
$$

for all $p$ and $q$ with $(h(i)-1) \cdot 2^{n}+1 \leqslant p<(h(i)) \cdot 2^{n}$ and $1 \leqslant q<$ $(2 i+1) \cdot 2^{n}$ (see Figure 2), hence

$$
\star \mathfrak{T}_{\overline{0}_{p}}^{\overline{0}_{p+1}} \equiv_{n} \star \mathfrak{T}_{\overline{0}_{(h(0)-1) \cdot 2^{n}}}^{\overline{0}_{(h(0)-1) \cdot 2^{n}} \overline{1}_{q+1}}
$$

for all such $p$ and $q$. Let

$$
\begin{aligned}
\mathfrak{L} & =\left(\left[\overline{0}_{(h(i)-1) \cdot 2^{n}+1}, a_{i}\right) ;<\Gamma_{\left[\overline{0}_{(h(i)-1) \cdot 2^{n}+1}, a_{i}\right)}\right), \\
\mathfrak{M} & =\left(\left[\overline{0}_{(h(0)-1) \cdot 2^{n}} \overline{1}_{1}, b_{i}\right) ;<\Gamma_{\left[\overline{0}_{(h(0)-1) \cdot 2^{n}} \overline{1}_{1}, b_{i}\right)}\right) .
\end{aligned}
$$

Note that $\|\mathfrak{L}\|,\|\mathfrak{M}\| \geqslant 2^{n}-1$ so by (2), $\mathfrak{L} \equiv_{n} \mathfrak{M}$. Since there are $\|\mathfrak{L}\|$ many trees of the form ${ }_{\star} \mathfrak{T}_{\overline{0}_{p}}^{\overline{0}_{p+1}}$, and $\|\mathfrak{M}\|$ many trees of the form $\star^{\mathfrak{T}^{0}} \overline{\overline{0}}_{(h(0)-1) \cdot 2^{n} \overline{1}_{q+1}}$, then it follows from Lemma 5 that

$$
{ }^{T^{2}} \overline{0}_{(h(i)-1) \cdot 2^{n}+1}^{a_{i}} \equiv_{n} \quad \mathfrak{T}_{\overline{0}_{(h(0)-1) \cdot 2^{n}} \overline{1}_{1}}^{b_{i}} .
$$

Proof of (7): This follows from the fact that $\mathfrak{T}_{a_{i}} \cong \mathfrak{T}_{b_{i}}$. 

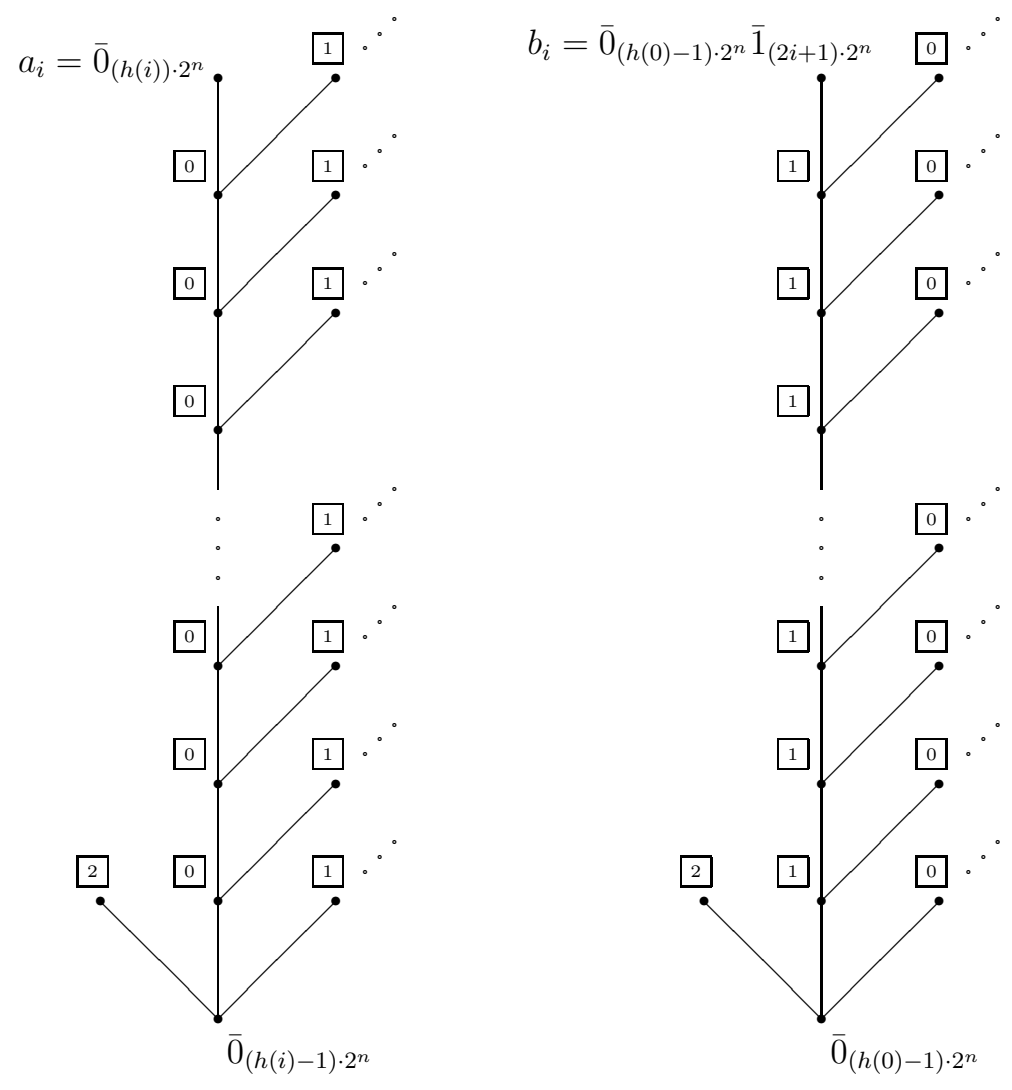

Figure 2: The trees ${ }_{\star} \mathfrak{T}_{\overline{0}_{(h(i)-1) \cdot 2^{n}}^{a_{i}}}$ (left) and ${ }_{\star} \mathfrak{T}_{\overline{0}_{(h(0)-1) \cdot 2^{n}}^{b_{i}}}$ (right) used in the proofs of (5) and (6) in Example 16. Function values under $f$ are indicated in boxes.

Hence we conclude that $\left(\mathfrak{T} ; a_{i}\right) \equiv_{n}\left(\mathfrak{T} ; b_{i}\right)$. Now to see that $(\mathfrak{T} ; A) \not_{n}$ $(\mathfrak{T} ; B)$, consider the following formulas:

$$
\begin{gathered}
\varphi(x):=\exists x_{1} \exists x_{2} \exists x_{3}\left(\wedge_{i \neq j}\left(x_{i} \neq x_{j}\right) \wedge \wedge_{i=1}^{3}\left(x<x_{i}\right) \wedge\right. \\
\left.\neg \exists z\left(\vee_{i=1}^{3}\left(x<z \wedge z<x_{i}\right)\right)\right) \\
\psi(X):=\forall x(X(x) \rightarrow \exists y(X(y) \wedge x<y \wedge \varphi(y)))
\end{gathered}
$$

The formula $\varphi(x)$ states that $x$ has three immediate successors and $\psi(X)$ states that every node in $X$ sits below another node in $X$ that has three immediate successors. Note that $\operatorname{qr}(\psi) \leqslant 6 \leqslant n$. Now $(\mathfrak{T} ; A) \models \psi(A)$ but $(\mathfrak{T} ; B) \not \forall \psi(B)$ hence $(\mathfrak{T} ; A) \not_{n}(\mathfrak{T} ; B)$. This concludes our counterexample to the property $\mathrm{CP}$.

Call a cover $\left(a_{i}\right)_{i \in \alpha}$ of a tree $(\mathfrak{T} ; A)$ an $n$-elementary cover when ${ }_{\star} \mathfrak{T}^{a_{i}} \preceq_{n} \star_{\mathfrak{T}^{a_{j}}}$ for all $i$ and $j$ with $i<j$. Note that if $\left(a_{i}\right)_{i \in \alpha}$ is an 
$n$-elementary cover of $(\mathfrak{T} ; A)$ then no node $a_{i}$ can have an immediate predecessor: if $b$ were an immediate predecessor of $a_{i}$ then

$$
\left(\star^{\mathfrak{T}^{a_{i+1}}} ; b\right) \models \exists x\left(b<x \wedge\left(a_{i+1}\right)^{>}(x)\right)
$$

while

$$
\left({ }_{\star} \mathfrak{T}^{a_{i}} ; b\right) \not \models \exists x\left(b<x \wedge\left(a_{i}\right)^{>}(x)\right),
$$

a contradiction with the fact that ${ }_{\star} \mathfrak{T}^{a_{i}} \preceq_{n} \star_{\mathfrak{T}^{a_{i+1}}}$. Hence well-founded trees $(\mathfrak{T} ; A)$ of height less than $\omega^{2}$ cannot have $n$-elementary covers.

While Example 16 shows that the cover property $\mathrm{CP}$ does not hold, a sufficient condition for the conclusion ${ }_{\star} \mathfrak{T}^{A} \equiv_{n}{ }_{\star} \mathfrak{S}^{B}$ of CP to hold is that the covers $\left(a_{i}\right)_{i \in \alpha}$ and $\left(b_{i}\right)_{i \in \alpha}$ be $n$-elementary covers.

Proposition 17 Let $\left(a_{i}\right)_{i \in \alpha}$ and $\left(b_{i}\right)_{i \in \alpha}$ be n-elementary covers for the trees $(\mathfrak{T} ; A)$ and $(\mathfrak{S} ; B)$ respectively. If $\left(\mathfrak{T} ; a_{i}\right) \equiv_{n}\left(\mathfrak{S} ; b_{i}\right)$ for every $i$ then ${ }_{\star} \mathfrak{T}^{A} \equiv_{n \star} \mathfrak{S}^{B}$.

Proof Let $\left(a_{i}\right)_{i \in \alpha}$ and $\left(b_{i}\right)_{i \in \alpha}$ be $n$-elementary covers for $(\mathfrak{T} ; A)$ and (S; $B)$. Then

$$
\begin{aligned}
& { }_{\star} \mathfrak{T}^{a_{k}} \preceq_{n} \bigcup_{i \in \alpha}{ }_{\star} \mathfrak{T}^{a_{i}}={ }_{\star} \mathfrak{T}^{A} \text { and } \\
& { }_{\star} \mathfrak{S}^{b_{k}} \preceq_{n} \bigcup_{i \in \alpha}{ }_{\star} \mathfrak{S}^{b_{i}}={ }_{\star} \mathfrak{S}^{B}
\end{aligned}
$$

for all $k$. Furthermore, since $\left(\mathfrak{T} ; a_{i}\right) \equiv_{n}\left(\mathfrak{S} ; b_{i}\right)$ for every $i$ then it follows that ${ }_{\star} \mathfrak{T}^{a_{k}} \equiv_{n \star} \mathfrak{S}^{b_{k}}$ too. Hence ${ }_{\star} \mathfrak{T}^{A} \equiv_{n \star} \mathfrak{S}^{B}$.

\section{Acknowledgements}

The author is grateful for the valuable suggestions made by an anonymous referee.

\section{References}

[1] R. Backofen, J. Rogers and K. Vijay-Shankar, A First-Order Axiomatization of the Theory of Finite Trees, Journal of Logic, Language and Information, vol. 4 (1995), pp. 5-39.

[2] K. Doets, Completeness and Definability: Applications of the Ehrenfeucht Game in Second-Order and Intensional Logic, Ph.D. thesis, University of Amsterdam, 1987. 
[3] K. Doets, Basic Model Theory, CSLI Publications \& The European Association for Logic, Language and Information, 1996.

[4] S. Feferman and R.L. Vaught, The First Order Properties of Products of Algebraic Systems, Fundamenta Mathematicae, vol. 47 (1959), pp. 57-103.

[5] A. Gheerbrant and B. Ten Cate, Complete Axiomatizations of $M S O, F O\left(T C^{1}\right)$ and $F O\left(L F P^{1}\right)$ on Finite Trees, Proceedings of $\boldsymbol{L F C S}$ 2009, S. Artëmov and A. Nerode (editors), Springer, 2009, pp. 180-196.

[6] V. Goranko, Trees and Finite Branching, Proceedings of the 2nd Panhellenic Logic Symposium, July 13-17, 1999, Delphi, Greece, P. Kolaitis and G. Koletsos (editors), National Technical University of Athens, 1999, pp. 91-101.

[7] V. Goranko and R. Kellerman, Classes and Theories of Trees Associated with a Class of Linear Orders, Logic Journal of the IGPL, vol. 19 (2011), pp. 217-232.

[8] V. Goranko and A. Zanardo, From Linear to Branching-Time Temporal Logics: Transfer of Semantics and Definability, Logic Journal of the IGPL, vol. 15 (2007), pp. 53-76.

[9] Y. Gurevich, Monadic Second-Order Theories, ModelTheoretical Logics, J. Barwise and S. Feferman (editors), Springer, 1985, pp. 479-506.

[10] F. Mwesigye and J.K. Truss, Classification of Finite Coloured Linear Orderings, Order, vol. 28 (2011), pp. 387-397.

[11] M.O. Rabin, Decidability of Second-Order Theories and Automata on Infinite Trees, Transactions of the American Mathematical Society, vol. 141 (1969), pp. 1-35.

[12] J.G. Rosenstein, Linear Orderings, Academic Press, 1982.

[13] J.H. Schmerl, On $\aleph_{0}$-Categoricity and the Theory of Trees, Fundamenta Mathematicae, vol. 94 (1977), pp. 121-128.

[14] S. Shelah, The Monadic Theory of Order, Annals of Mathematics, vol. 102 (1975), pp. 379-419. 